\title{
ASSESSING THE ASSOCIATION BETWEEN CARE QUALITY AND HOUSEHOLD CHORE RULES IN RESIDENTIAL CARE INSTITUTIONS IN JAPAN
}

\section{Rie Mizuki, Mamiko Kyuzen, Satoru Nishizawa, and Shigeyuki Mori}

\begin{abstract}
The performance of household chores by children in Japanese residential care institutions has been widely accepted as a practice that fosters children's independence and self-sufficiency. However, children coming from neglectful or dysfunctional families often require sensitive, individualized care, which they did not receive from their family of origin. While a shift away from large-scale institutions has begun, with smaller units or group homes now accounting for $40 \%$ of all care institutions, it is not clear that family-like, individualized care has been achieved in these smaller group homes. This study involved 61 participants aged 10 to 15 years and their care workers from six residential care institutions in the greater Tokyo area. It explored what aspects of care quality were related to the presence of certain characteristics of traditional management-oriented care: fixed rules for household chores and lack of choice in daily living. Care workers were asked whether children washed their own dishes, did their own laundry, chose their own clothes when shopping, and had mandatory events to attend in the residential care institutions. Care quality was assessed using the Early Adolescent HOME (Home Observation for Measurement of Environment) developed for Japanese residential care institutions (EA-HOME-JP). The findings suggest that care workers should re-examine their intentions in setting and applying household chore rules while exploring which aspects of child care, including regulatory activities and modeling, should be emphasized in order to actualize family-like and individualized care in institutions.
\end{abstract}

Keywords: HOME, institutional rules, residential care, alternative care

Rie Mizuki MA (corresponding author) is a Lecturer at the Radiation Medical Science Center for the Fukushima Health Management Survey, Fukushima Medical University, 1 Hikariga-oka, Fukushima City 960-1295, Japan. Email: mizu5656@ hotmail.com

Mamiko Kyuzen MA is a Clinical Psychologist at Aisenryo, 2-15-57 Dote, Kazo, Saitama, 3478510, Japan.

Satoru Nishizawa MS is a Professor in the Faculty of Human and Social Services, Yamanashi Prefectural University, 5-11-1 Iida, Kofu, Yamanashi, 400-0035, Japan.

Shigeyuki Mori PhD is a Professor in the Department of Human Sciences, Faculty of Letters, Konan University, 8-9-1 Okamoto, Higashi-Nada, Kobe, Hyogo, 658-8501, Japan. 
Rules and tasks have traditionally been used in Japanese residential care institutions as tools to manage and regulate children's daily activities and behaviors. The lives of children at these institutions have usually been structured using a daily routine timetable consisting of a series of chores and tasks, all of which the children are expected to follow. In interviews, care staff and children placed in these institutions during the 1950s and 1960s have described the typical routine: a fixed morning wake-up time, cleaning one's own bedroom, taking turns to clean common areas, a time for morning physical exercise, washing one's own used lunch box, eating a snack, and taking a bath every other day on a predetermined schedule (Kikuchi, 1993). Older children were also expected to take turns washing everyone's dishes after breakfast and assisting in the bathing of younger children. Similar routines were revealed in the case analysis of an institution located in Tokyo during the 1940s and 1950s (Kikuchi \& Watanabe, 1995). After waking up at a fixed time, children took turns in cleaning designated areas, washing dishes, carrying and chopping firewood, and assisting in cooking meals. Having such a regimented structure was considered important in fostering a sense of predictability and stability in everyday life, conditions that many of those children's lives might have lacked prior to their placement. The tradition of rules setting, by which children are expected to perform assigned chores, survived into the second half of the 20th century: a study in 1998 showed that $79.5 \%$ of the children at institutions were assigned tasks and roles (as cited in Hayashi, 2000).

Children's dissatisfaction with the lack of freedom of choice in their daily lives and strict or unreasonable application of institutional rules was uncovered by recent qualitative studies. In Ito's 2010 study, in which 10 children living in a residential care institution were interviewed about their daily lives, the children reported their dissatisfaction with the rigid schedule, which stipulated preset meal and bath-time schedules and a curfew. Some of their complaints were:

We aren't allowed to have a meal a little later than the scheduled time based on how we feel or our physical conditions on that day.... Dinner time is set too early, so we become hungry at night.... We are told to "take a bath between X o'clock and Y o'clock." We cannot take a bath when we like... The curfew of six o'clock is too early. (p. 87)

Constraints in other domains were also observed in the same study:

[We are told that wearing clothes with] a design of a skull is not okay, leopard print is not okay. We don't have freedom of buying our own clothes.... We cannot ride a bike without permission, and there's an age limitation, and also a bicycle exam.... Video games are banned, so we can't play even for one minute, and only card games are permitted. (p. 87)

Institutions sometimes procure children's clothes by blanket purchase for the entire institution; such practices not only fail to attend to each child's needs and preferences, they take away 
opportunities in which children could enjoy the process of shopping and choosing clothes through positive communication with care workers (Nagai, 2006). Similar reports of dissatisfaction, such as institutions restricting hobbies like computer games and banning ear piercings and dyed hair, were observed among the adolescents in Fujita's 2012 study (p. 183). In another study, Taniguchi (2018) found that fixed rules were applied strictly to all children regardless of the individual child's age or needs; these included: not permitting a romantic relationship with a child living in the same institution; not permitting children to spend money earned through part-time jobs, instead forcing them to save it in their bank accounts; not allowing children to own cell phones unless the child's parent agreed to pay; and requiring that, if they purchased shampoo or soap of their choice, it must be paid for from their allowance. Thus, growing up in an institution involves being bound by responsibility for tasks and chores, alongside adherence to stringently defined schedules and rules (Nagase \& Karino, 2012).

Fixed rules were and are adopted by many institutions in Japan in order to provide structure to daily living where many children live together (Taniguchi, 2018). Kato (2003) pointed out that care workers, given their high workloads, could not help but prioritize the efficient management of children; they accomplish this by establishing a detailed daily schedule and enforcing strict rules under the name of communal living. Thus the rules are for the benefit of alleviating the high work demands of care workers when managing a large number of children. Further, each child's needs or requests may be neglected or subjugated in an attempt to ensure "equality" in the treatment of children, which in turn could hinder the growth of children's autonomy and independence (Kato, 2003). Nonetheless, the above quotations exemplify how rigidly rules were implemented and enforced, and how constrained the children might be feeling in various aspects of their lives in the institution. While such conditions in Japanese institutions - the lack of reasonable amounts of flexibility and individualized treatment — do not indicate severely deprived environments or poor care quality, they do signify that the children experience challenges derived from communal living in large groups, challenges that children living with their parents at home rarely face (Hirata \& Negayama, 2012). Thus, rigid rules may impede efforts to provide more individualized and familylike care for children living in institutions.

With the global trend moving in the direction of providing a therapeutic environment and sensitive, individualized care to children in alternative care, the Japanese government has been introducing financial incentives to abolish large-scale residential care institutions in favor of smaller residential facilities that can provide a family-like environment. Such measures have resulted in the adoption of smaller-scale residences that house between six and eight children, with a single bedroom (in principle) for each child. These residences are each equipped with a common bathroom, and a kitchen where care workers cook for the children. Small-scale residences are structured either as an independent house (group home) or apartment-style in a building (small unit). There are also medium-sized units with 13 to 19 children and large-sized units with 20 children or more. The bigger units have a kitchen where cooking staff fix food for all the children 
International Journal of Child, Youth and Family Studies (2020) 11(4): 80-95

and one large dining room in which all eat together; bedrooms are usually shared by two to four children.

These smaller-scale residences now account for approximately $40 \%$ of residential care facilities (Ministry of Health, Labour and Welfare, 2019). The steps to achieving family-like and individualized care, similar to the experiences of children living with their family, are described by the Ministry of Health, Labour, and Welfare (2019) as: teaching household chores through sharing everyday life, providing home cooking, establishing a less stressful environment to foster greater emotional stability for the children, and developing a sense of home life so that children may envision having families of their own in the future. However, these care requirements cannot be met by simply reducing the scale of the residential environment while continuing to practise the type of management-oriented traditional care that applies uniform rules to all children and does not take into consideration each child's individual needs.

Even where rigid adherence to rules and tasks is still maintained, in some cases care workers have been able to creatively apply these rules to daily living. For example, in some institutions, care workers chose to do laundry for the children; they felt it was important for the children to experience being cared for by adults, since many came from family environments in which such ordinary care was not often provided (Taniguchi, 2018). Some children lack knowledge of basic self-care practices, such as how to take a bath, how to wash their bodies, or how often to change clothing to avoid wearing soiled garments; for those children to master these basic skills, it is necessary for care workers to customize their approach, including engaging in washing and bathing the children, dressing them in clean clothes, and folding their dried laundry with them, instead of just instructing them to take a bath or wash their clothes (Sato et al., 2011). Mandatory tasks, often prescribed as rules, may impede the development of children's independence if they have not had the experience during their early childhood of being completely dependent on and cared for by adults (Nagai, 2006). The above examples of caregiving go beyond the traditional stance of Japanese residential care institutions, which focus on the group management of children, to consider children's individual and developmental needs as well. Thus, some residential units appear to be moving toward the individualized and family-like care model proposed by the Ministry.

Studies in smaller children's homes reported greater emotional closeness (Iida, 2010), increased communication between care worker and child, more individualized treatment (Yoshimura \& Yoshimura, 2016), and greater flexibility in daily routines through eliminating certain rules that were being applied to all children (Gonokama, 2013). Although these positive changes may derive from downsizing the residential units, discrepancies were nevertheless observed between care workers in the way they perceived children's ability to become selfsufficient and in the extent to which they incorporated children's opinions into daily rituals (Kobe, 2007). Although the downsizing of residential units may impact care practices, including the strict and universal application of daily routines and institutional rules, research evaluating the 
International Journal of Child, Youth and Family Studies (2020) 11(4): 80-95

relationship between rule implementation and care quality in residential institutions remains scarce.

The purpose of the current study, therefore, was to explore the possible impact on care quality of traditional rules for chores (e.g., washing one's own dishes and doing one's own laundry), and rules for limiting choice in daily living (e.g., not being allowed to purchase clothes of one's choosing and having mandatory events to attend), which are still implemented at many residential institutions in Japan. This study is a part of the research project in which the Japanese version of Home Observation and Measurement of Environment-Early Adolescent version (EA-HOME-JP; Zhang et al., 2018) was introduced in order to objectively measure care quality in residential care institutions in the greater Tokyo area.

The EA-HOME-JP was developed and tailored for Japanese residential care institutions in order to assess the "soft" dimension of care giving (Zhang et al., 2018). In the preliminary results of their study, the scale of residential unit was not associated with care quality, implying that the reduction in residential unit size does not automatically mean that the quality of care improves or that family-like care is achieved. Thus, the impact of a smaller residential unit (the "hard" dimension of institutional care) on the quality of care practices (the "soft" dimension) needs to be further examined.

The EA-HOME-JP study dataset was used for the analysis. Hence, this study explored the associations between the presence of traditional institutional rules (i.e., washing dishes, washing clothes, choosing their own clothes, and attending mandatory events) and the scores on various aspects of care quality measured with EA-HOME-JP.

\section{Method}

\section{Participants}

The sample consisted of 61 children, aged 10 to 15 years, and their care workers from six residential care institutions in the greater Tokyo area that comprised a mix of group homes, small units, and medium-sized units ${ }^{1}$. At each institution, the staff approached children in the target age range, introduced the study, and asked if they were interested in participating. If a child showed interest in the study, the care worker contacted the research team. If requested by care workers, the research team members visited the institution and explained the details of the study to those children - individually in some institutions and in a group format in others — so that the children could ask questions of the research team directly. After confirming the children's interest in taking part in the study, care workers contacted the research team and the timing of an interview was arranged, based on the child's availability. Participants thus comprised the children who both

\footnotetext{
${ }^{1}$ For instance, one institution in the study owned a large building containing six small residential units on the premises and three external group homes. Another institution owned a large building containing one medium unit on the premises and three external group homes.
} 
International Journal of Child, Youth and Family Studies (2020) 11(4): 80-95

consented and were available for the interview. At the data collection session, a research team member again explained the study, as detailed in the ethics section below. Written assent from each child participant was then collected. Two cases were excluded due to missing demographic data, resulting in 59 children being included in the analyses.

Table 1. Sample Characteristics $(N=59)$

\begin{tabular}{lcccc}
\hline Characteristic & $n$ & $\%$ & $M$ & $S D$ \\
\hline Age & & & 12.65 & 1.49 \\
Sex & & & & \\
$\quad$ Boy & 30 & 50.9 & & \\
$\quad$ Girl & 29 & 49.1 & & \\
Residential type & & & & \\
$\quad$ Group home & 16 & 27.1 & & \\
$\quad$ Small unit & 30 & 50.9 & & \\
$\quad$ Medium-sized unit & 13 & 22.0 & & \\
Current placement in months & & & 89.2 & 46.15 \\
Total placement in months & & & 100.7 & 47.33 \\
No parental consent & 3 & 5.08 & & \\
$\quad$ No communication with parents & 18 & 30.5 & & \\
$\quad$ No meeting with parents & 16 & 27.1 & & \\
$\quad$ Nursery placement & 23 & 39.0 & & \\
Maltreatment experiences & & & & \\
$\quad$ Physical abuse & 21 & 37.5 & & \\
$\quad$ Emotional abuse & 14 & 25.0 & & \\
$\quad$ Sexual abuse & 2 & 3.6 & & \\
$\quad$ Neglect & 25 & 43.9 & & \\
\hline
\end{tabular}

As shown in Table 1, 50.9\% of the child participants were boys. Almost half had been in placement for 8 years or longer. A slight majority (50.9\%) of the children were living in small units that were equipped with a private kitchen and bathroom, in addition to bedrooms that accommodated 6 to 8 children. In some cases, the small units were housed within a large institution. Sixteen $(27.1 \%)$ of the children lived in group homes of up to six children each; most were houses located in residential communities close to the main premises of the parent institution. Thirteen (22.0\%) lived in medium-sized units, where common facilities such as bathrooms, dining room, and kitchen were usually shared with other units, accommodating 13 to 19 children.

Based on the reports by their care workers, many of the participating adolescents had experienced maltreatment: physical abuse (37.5\%), emotional abuse (25.0\%), sexual abuse (3.6\%), and neglect (43.9\%). The mean duration in which the children were in out-of-home care was over eight years $(M=100.72$ months, $S D=47.33)$. Considering that their mean age was 12.65 years, 
International Journal of Child, Youth and Family Studies (2020) 11(4): 80-95

these adolescents on average had spent two-thirds of their lives away from their parents. Furthermore, contact with their parents was hampered for many adolescents: $30.5 \%$ reported having no communication, including phone calls and letters, and $27.1 \%$ had no direct meetings with their parents, such as going out for meals or sleeping over at their parent's residence. While the parents of most of the children agreed to place their child in an institution, for $3 \%$ of the children, parental consent could not be obtained and the children were in placement under the Child Welfare Act, Article 28, of Japan.

\section{Ethics}

The ethics committee at the Yamanashi Prefectural University approved this study. The heads of each institution, who have custody of the placed children, gave informed written consent for each child participant and the participating care workers. Written assent was also collected from all child participants. The research staff clearly explained to each child participant that: (a) their participation was completely voluntary; (b) all of their responses, except for the HOME interview, would be kept confidential, even from the staff at the institution; (c) they could withdraw from the study at any time; (d) no disadvantages would be associated with their refusal to participate or with their withdrawal from participation; and (e) the data included in the study reports could not be used to identify them. A number was assigned to each participant. The data were linked with these numbers and kept separate from the participants' personal information.

\section{Procedure}

One or two members of the research team visited each institution. They first met with each child individually and had the child fill out the questionnaire packet. The researchers then conducted an interview about care quality with both the child and his or her care worker. At the end of the interview, with the child's permission, the researcher visited the residential unit and the child's bedroom for observation. In the case of children who did not agree to the researchers' visit to their residential unit, the researchers asked the children to describe their current home in as much detail as possible. Participating care workers also filled out a questionnaire packet prior to the data collection. The child's and the care worker's responses to the questionnaire were collected separately and kept confidential.

\section{Materials: Care Quality}

Care quality was gauged using the Home Observation for Measurement of Environment for Early Adolescent (EA-HOME-JP), which was developed specifically for Japanese residential care institutions (Zhang et al., 2018). The measure comprises 64 items, answered with dichotomous ratings (yes $=1$, no $=0$ ). The items contain seven subscales:

1. Physical environment, measuring safety, space, and age appropriateness of the physical environment (e.g., Adolescent's room has at least two pictures or decorations appealing to an adolescent; House or apartment has no potentially dangerous structural or health hazards); 
International Journal of Child, Youth and Family Studies (2020) 11(4): 80-95

2. Learning materials, assessing resources and conditions available for children's learning (e.g., Adolescent has access to at least 20 developmentally appropriate books; Adolescent has access to home computer);

3. Modeling, evaluating whether or not the caregiver functions as a positive role model (e.g., Care worker shares healthy practices/fitness activities that s/he regularly engages in; Care worker has not lost their temper with an adolescent more than once during the past week);

4. Fostering self-sufficiency, indicating how the caregiver integrates activities promoting children's self-sufficiency (e.g., Care worker teaches adolescent basic cleaning skills; Care worker has taught adolescent about safety and how to deal with emergency situations);

5. Regulatory activities, representing regulations and supervision set by care workers as caring and responsible caregivers (e.g., Residential unit has a fairly regular and predictable daily schedule; Parent has had contact with at least 2 of the adolescent's friends in the last month);

6. Family companionship, rating the provision of social and cultural activities, family-like atmosphere, and fair relationships among children (e.g., Care worker regularly engages in outdoor activity with the adolescent at least once every two weeks; Children interact with both male and female care workers at least four days a week); and

7. Acceptance, looking into whether there is a positive relationship between the care worker and child, including warm and caring attitudes of care workers (e.g., Care worker shows some positive emotional response when visitor praises adolescent; Care worker encourages adolescent to contribute to the conversation during visit).

Each subscale score was calculated by adding up the scores of corresponding items. The Cronbach alpha for each subscale is shown in Table 2.

Table 2. Cronbach's Alpha for Each Subscale

\begin{tabular}{lcc}
\hline Subscale & $\begin{array}{c}\text { No. of } \\
\text { items }\end{array}$ & $\alpha$ \\
\hline Physical environment & 7 & .50 \\
Modeling & 9 & .45 \\
Fostering self-sufficiency & 8 & .56 \\
Regulatory activities & 6 & .47 \\
Family companionship & 10 & .48 \\
Acceptance & 9 & .87 \\
\hline
\end{tabular}

Note. The learning materials subscale was excluded from the analyses due to low internal consistency. 
International Journal of Child, Youth and Family Studies (2020) 11(4): 80-95

\section{Materials: Institutional Rules}

Care workers answered yes/no questions regarding rules for tasks and household chores. The following questions were included:

1. Do children at your home wash their own dishes due to an institutional rule?

2. Are children at your home supposed to do their own laundry based on an established rule?

3. Can children at your home choose their own clothes when shopping? and

4. Do children at your home have to attend mandatory events planned by the institution?

\section{Analysis}

First, a Kruskal-Wallis rank test was conducted for the EA-HOME-JP subscale scores by residential type. Second, a correlation analysis examined the relationships among the subscale scores. Third, bivariate and multivariate logistic regressions were conducted to examine whether or not there were associations between the subscale scores and institutional rules. All statistical analyses were conducted with STATA (version 13.1; Stata Corp College Station, Texas, United States). The level of statistical significance was set at $p<.05$ (two-tailed).

\section{Results}

The distribution of EA-HOME-JP subscales by residential types is presented in Table 3. Only family companionship $\left(\chi^{2}[2]=6.73, p<.05\right)$ and acceptance $\left(\chi^{2}[2]=12.0, p<.01\right)$ were impacted by residential type; significant median score differences among the residential types were observed. A post-hoc Dunn test revealed that medium-sized units (median $=5.0$ ) had a significantly lower score in family companionship than small units (median $=7.0$ ). In acceptance, medium-sized units scored significantly lower than either group homes (median $=9.0$ ) or small units (median =9.0). Marginally significant differences were found in fostering self-sufficiency $\left(\chi^{2}[2]=4.88, p<.10\right)$ and regulatory activities $\left(\chi^{2}[2]=5.837, p<.10\right)$. There were no differences across the residential types in the scores for physical environment and modeling. 
International Journal of Child, Youth and Family Studies (2020) 11(4): 80-95

Table 3. EA-HOME-JP Subscale Score Distributions by Residential Type

\begin{tabular}{|c|c|c|c|c|c|c|c|c|c|c|}
\hline \multirow[b]{2}{*}{ Subscale } & \multicolumn{3}{|c|}{ Group homes $(n=16)$} & \multicolumn{3}{|c|}{ Small units $(n=30)$} & \multicolumn{3}{|c|}{ Medium units $(n=13)$} & \multirow[b]{2}{*}{$p^{*}$} \\
\hline & Mdn & $25 \%$ & $75 \%$ & Mdn & $25 \%$ & $75 \%$ & Mdn & $25 \%$ & $75 \%$ & \\
\hline Physical environment & 7.00 & 7.00 & 7.00 & 7.00 & 6.00 & 7.00 & 6.00 & 6.00 & 7.00 & 0.303 \\
\hline Modeling & 6.50 & 5.50 & 8.00 & 6.00 & 5.00 & 7.00 & 6.00 & 5.00 & 6.00 & 0.125 \\
\hline Fostering self-sufficiency & 6.00 & 4.50 & 7.75 & 5.00 & 3.00 & 6.00 & 4.00 & 4.00 & 5.00 & 0.087 \\
\hline Regulatory activities & 7.00 & 5.50 & 7.00 & 6.00 & 5.00 & 7.00 & 7.00 & 7.00 & 7.00 & 0.054 \\
\hline Family companionship & 6.00 & 4.50 & 7.00 & 7.00 & 6.00 & 8.00 & 5.00 & 4.00 & 6.00 & 0.035 \\
\hline Acceptance & 9.00 & 9.00 & 9.00 & 9.00 & 9.00 & 9.00 & 7.00 & 7.00 & 8.00 & 0.003 \\
\hline
\end{tabular}

* probability for Kruskal-Wallis rank test.

Table 5. Bivariate Odds Ratio of Institutional Rules by EA-HOME-JP Subscale Score

\begin{tabular}{|c|c|c|c|c|c|c|c|c|}
\hline \multirow[b]{2}{*}{ Subscale } & \multicolumn{2}{|c|}{ Wash dishes $(n=56)$} & \multicolumn{2}{|c|}{ Do laundry $(n=59)$} & \multicolumn{2}{|c|}{ Choose clothes $(n=59)$} & \multicolumn{2}{|c|}{ Attend event $(n=59)$} \\
\hline & $O R$ & $95 \% \mathrm{CI}$ & $O R$ & $95 \% \mathrm{CI}$ & $O R$ & $95 \% \mathrm{CI}$ & $O R$ & $95 \% \mathrm{CI}$ \\
\hline Physical environment & 1.04 & {$[0.51,2.10]$} & 1.55 & {$[0.75,3.21]$} & $\mathrm{n} / \mathrm{a}$ & & 0.84 & {$[0.47,1.52]$} \\
\hline Modeling & 0.69 & {$[0.40,1.20]$} & $0.52 *$ & {$[0.25,1.08]$} & 1.20 & {$[0.61,2.37]$} & 0.80 & {$[0.51,1.26]$} \\
\hline Fostering self-sufficiency & 1.21 & {$[0.85,1.73]$} & 1.36 & {$[0.90,2.07]$} & 1.02 & {$[0.67,1.55]$} & 0.78 & {$[0.58,1.05]$} \\
\hline Regulatory activities & $2.94 * *$ & {$[1.46,5.91]$} & $3.74 * *$ & {$[1.52,9.17]$} & 1.06 & {$[0.57,1.97]$} & 0.93 & {$[0.62,1.42]$} \\
\hline Family companionship & 1.05 & {$[0.74,1.49]$} & 1.42 & {$[0.92,2.20]$} & 1.10 & {$[0.71,1.69]$} & 1.05 & {$[0.79,1.40]$} \\
\hline Acceptance & 0.50 & {$[0.18,1.40]$} & $\mathrm{n} / \mathrm{a}$ & & 1.01 & {$[0.66,1.55]$} & 0.79 & {$[0.57,1.09]$} \\
\hline
\end{tabular}

$* \mathrm{p}<.10, * * \mathrm{p}<.05$.

Table 6. Multivariate Odds Ratio of Institutional Rules by EA-HOME-JP Subscale Score

\begin{tabular}{|c|c|c|c|c|c|c|c|c|}
\hline \multirow[b]{2}{*}{ Subscale } & \multicolumn{2}{|c|}{ Wash dishes $(n=56)$} & \multicolumn{2}{|c|}{ Laundry $(n=44)$} & \multicolumn{2}{|c|}{ Choose clothes $(n=39)$} & \multicolumn{2}{|c|}{ Attend event $(n=59)$} \\
\hline & $O R$ & $95 \% \mathrm{CI}$ & $O R$ & $95 \% \mathrm{CI}$ & $O R$ & $95 \% \mathrm{CI}$ & $O R$ & $95 \% \mathrm{CI}$ \\
\hline Physical environment & 0.75 & {$[0.28,1.97]$} & 1.48 & {$[0.39,5.58]$} & $\mathrm{n} / \mathrm{a}$ & & 1.01 & {$[0.51,2.03]$} \\
\hline Modeling & 0.72 & {$[0.36,1.44]$} & $0.35^{*}$ & {$[0.12,1.02]$} & 1.13 & {$[0.56,2.28]$} & 0.91 & {$[0.55,1.50]$} \\
\hline Fostering self-sufficiency & 1.19 & {$[0.64,2.21]$} & 0.94 & {$[0.40,2.17]$} & 1.16 & {$[0.62,2.18]$} & 0.72 & {$[0.47,1.09]$} \\
\hline Regulatory activities & $2.78 * *$ & {$[1.29,6.00]$} & $4.50 * *$ & {$[1.16,17.53]$} & 1.23 & {$[0.58,2.62]$} & 1.02 & {$[0.63,1.66]$} \\
\hline Family companionship & 0.96 & {$[0.55,1.66]$} & 1.70 & {$[0.74,3.89]$} & 1.15 & {$[0.70,1.88]$} & 1.26 & {$[0.87,1.82]$} \\
\hline Acceptance & 0.55 & {$[0.18,1.66]$} & $\mathrm{n} / \mathrm{a}$ & & 1.03 & {$[0.62,1.72]$} & 0.85 & {$[0.61,1.18]$} \\
\hline
\end{tabular}

$* \mathrm{p}<.10, * * \mathrm{p}<.05$. 
International Journal of Child, Youth and Family Studies (2020) 11(4): 80-95

The Spearman correlation analyses (see Table 4) revealed low to moderate correlations between some subscales of EA-HOME-JP. Physical environment was significantly positively correlated with fostering self-sufficiency $\left(r_{s}=.38, p<.05\right)$. Also, modeling was significantly positively correlated with acceptance $\left(r_{s}=.28, p<.05\right)$. Positive correlations of fostering selfsufficiency with regulatory activities $\left(r_{s}=.31, p<.05\right)$ and family companionship $\left(r_{s}=.44, p<.05\right)$ reached statistical significance. Despite this, the lack of high correlation coefficients indicated that the correlations of these subscale scores were not strong. It is suggested that the subscales measured different concepts, even though some subscales might represent somewhat overlapping components of the caring environment.

Table 4. Correlation Coefficients Among EA-HOME-JP Subscale Scores

\begin{tabular}{lccccc}
\hline Subscale & 1 & 2 & 3 & 4 & 5 \\
\hline 1. Physical environment & 1.00 & & & & \\
2. Modeling & 0.13 & 1.00 & & & \\
3. Fostering self-sufficiency & $0.38^{*}$ & 0.26 & 1.00 & & \\
4. Regulatory activities & 0.20 & -0.18 & $0.31^{*}$ & 1.00 & \\
5. Family companionship & 0.23 & 0.14 & $0.44^{*}$ & -0.03 & 1.00 \\
6. Acceptance & 0.07 & $0.28^{*}$ & 0.25 & -0.19 & 0.08 \\
\hline
\end{tabular}

Note. Spearman correlation analysis was conducted.

$* p<.05$ level.

Bivariate logistic regressions revealed some associations between the EA-HOME-JP subscale scores and institutional rules, as shown in Table 5. EA-HOME-JP regulatory activities had significantly higher odds ratios $(O R)$ on washing dishes $(O R=2.94 ; 95 \%$ CI $[1.46,5.91])$ and doing laundry $(O R=3.74 ; 95 \% \mathrm{CI}[1.52,9.17])$. Residential units with higher scores on regulatory activities were more likely to direct children to wash their own dishes after meals and to do their own laundry by setting these tasks as rules. On the other hand, modeling had an odds ratio on doing laundry $(O R=0.52 ; 95 \%$ CI $[0.25,1.08])$ with higher significance in the protective direction, but only marginally so. This meant that the residential unit scoring higher on modeling tended not to let children do their own laundry; instead, those care workers tended to do laundry for their children. There were no significant associations between EA-HOME-JP subscale scores and the rule of mandatory attendance at institutional events.

With multivariate logistic regression, including all EA-HOME-JP subscale scores, regulatory activities was significantly associated with washing dishes $(O R=2.78,95 \%$ CI $[1.29,6.00])$ and with laundry $(O R=4.50,95 \%$ CI $[1.16,17.53])$, as presented in Table 6. Residential units with higher scores on regulatory activities were likely to have a rule directing their children to do dishes after the meal and their own laundry, after controlling for the influence of other subscales. Modeling maintained its marginal significance with an odds ratio of 0.35 (95\% CI [0.12, 1.02]) in the protective direction, on doing their own laundry. The care workers who scored higher on modeling are likely to take care of laundry for the children, instead of telling the children to do it 
International Journal of Child, Youth and Family Studies (2020) 11(4): 80-95

themselves, independent of the impact of other subscale scores. In multivariate models as well, none of the EA-HOME-JP subscale scores were associated with children's choosing their own clothes and mandatory attendance at institutional events.

\section{Discussion}

Using Japanese data from the greater Tokyo area, the current study examined the relationship between the quality of care as measured by EA-HOME-JP, and three types of residential care: group homes, small units, and medium-sized units. The type of residence was found to make a difference in family companionship and acceptance scores. Further analyses using logistic regressions revealed that higher scores on the EA-HOME-JP subscale of regulatory activities had a higher odds ratio for having a rule telling children to wash their own dishes after meals and do their own laundry. In contrast, higher scores on the subscale of modeling showed a tendency to be associated with a lower odds ratio for a rule directing children to do their own laundry.

Because rigid rules are considered to reflect more traditional approaches to child care in these institutions, the associations with the rules suggested that EA-HOME-JP regulatory activities may be aligned with traditional "professional" practices of institutions, which focus on boundary setting and supervision. With their much higher child-staff ratio, the traditional institutions could not help but emphasize the management of stable care quality and regulation of children's behavior. As a matter of fact, the EA-HOME-JP regulatory activities subscale includes an item asking if children are assigned personal tasks or chores, or ones that benefit the entire residential unit. This suggests that even the items in the inventory were skewed towards meeting collective demands and goals rather than those of the individual. However, this subscale also investigates care workers' behaviors, such as whether they have information on how the child spends time and keeps routines during days away from the institution, or whether they establish basic routines of everyday life, such as by setting fixed times for waking, eating meals, and going to bed, which can give children a sense of predictability. These actions are regarded as reflecting care workers' intention to regulate children's daily lives by organizing them with some type of routine. Stability and predictability are often referred to as essential components for building a safe environment for maltreated children (Barton et al., 2011). Gonokama (2013) gave examples that describe a sensitive balance between maintaining structure in daily life and incorporating flexibility. Differences in the intentions and attitudes behind care workers' actions may be difficult to identify using a quantitative scale such as EA-HOME-JP, but they may be measurable by the acceptance subscale, while regulatory activities focuses on care workers' efforts to create a safe environment through establishing some routines and boundaries. It is important to share some guidelines with care workers, particularly at the institutional level, so that the management of care does not rely on the individual care worker's discretion.

Modeling was marginally associated $(O R=0.52)$ with doing their own laundry. This subscale assesses whether or not care workers read books in front of the child, talked about their personal 
friends, discussed current events, and so on. Showing the child how to do chores was not included in the subscale. Modeling rather measures whether care workers share their own opinions and experiences with the children. In other words, it reflects how much care workers, while maintaining professional boundaries, share aspects of their personal lives with the children. The importance of personal openness when interacting with children is addressed in the field of social pedagogy (Mori, 2016). Although modeling may be a new concept in child welfare in Japan, it should be integrated into the training of care workers and recognized as a critical concept for homelike, therapeutic care. The fact that at least one in four children does not see or is not in contact with their parents denotes the absence of parents or caregiving adults in their lives. To encourage the healthy socialization and development of such children, care workers need to function as role models, becoming involved with the children at the personal level. This will not only help compensate for the children's insufficient exposure to a healthy image of caregivers, but will lead to residential care institutions becoming less management-oriented, more individualized, and providing more family-like care.

\section{Limitations}

Several limitations must be addressed. First, EA-HOME-JP has not yet been validated. Lower variability in the physical environment is congruent with the results of Zhang et al. (2018) and may be attributed to the application of the national standards for residential care institutions, which are set by the Ministry and that firmly regulate structural design and physical environment. On the other hand, when using EA-HOME-JP as a tool to compare residential care institutions with foster homes and family household environments, the subscale of physical environment may become meaningful. In addition, low internal consistencies for the subscales, as indicated by the low Cronbach alpha levels in the current study, call into question the validity of the observed associations and demand careful interpretation of the results. The Cronbach alphas in the current study were lower than those indicated by Zhang et al. (2018). Thus, EA-HOME-JP question items must be scrutinized by further statistical analyses, such as factor analysis, using a larger sample. Although its convergent validity was implied by Zhang et al. (2018), further examination of the validity of the EA-HOME-JP is necessary.

Second, the current study employed a small convenience sample, which may have hampered the detection of associations between care quality and institutional rules. Because of the sampling procedure, the interpretation and generalization of the results requires careful consideration. In particular, the study was conducted in Tokyo, where the metropolitan government has been implementing its own policies for improving care quality in residential care institutions. Financial assistance and the child-staff ratio tend to be more generous in Tokyo than in other prefectures; thus; policy differences across prefectures must be integrated into future analyses with a larger and more representative sample. Therefore, caution must be exercised when applying the results, even to residential care institutions in the greater Tokyo area. 
International Journal of Child, Youth and Family Studies (2020) 11(4): 80-95

Third, care workers' responses to the questionnaires may have been influenced by social desirability. Working in the field of child welfare, most of the care workers are likely to be aware of the shift towards individualized care and small-unit residences strongly encouraged by the Japanese government. As a result, they may feel hesitant to report having rules for tasks and chores that are assigned to all children at their institution.

\section{Conclusion}

The current study explored care quality in Japanese residential care institutions by focusing on the traditional management-oriented method of rules and tasks, which assigned household chores to children. The results show that care workers' efforts to structure children's daily routines were associated with rules directing children to do chores. On the other hand, it was revealed that care workers' efforts to function as role models may impact traditional attitudes in institutions that focus on managing children's behavior through rigid rules.

Achieving family-like or individualized care in any one institution may depend on which aspects of care quality are emphasized by that institution. If the values of individualized or familylike care quality are shared by the staff, and such aspects of care quality are discussed and emphasized in staff meetings and personnel training, this can eventually lead to the abolishment of the rigid rules that characterize management-oriented care in residential institutions, and lead to the provision of more individualized, child-centered care. Hence, additional analysis of household chore rules that addresses modeling performed by care workers is suggested. The fact that approximately 30,000 children, who may have maltreatment histories, are still being raised in residential care institutions highlights the importance of improving child care quality by incorporating individualized and family-like care to foster children's healthy development. To realize this, further research endeavors are also called for in order to provide evidence for links between care quality and the application of rules in residential care institutions.

\section{Acknowledgement}

This research was supported by a grant from the Japanese Society for Prevention of Child Abuse and Neglect. 
International Journal of Child, Youth and Family Studies (2020) 11(4): 80-95

\section{References}

Barton, S., Gonzalez, R., \& Tomlinson, P. (2011). Therapeutic residential care for children and young people: An attachment and trauma-informed model for practice. Jessica Kingsley.

Fujita, T. (2012). The good staff are those who learn from those who have a life experience in a children's home: The result of the questionnaire given to the children [In Japanese]. Kinjo Gakuin Daigaku Ronshu, 8(2), 180-192.

Gonokama, K. (2013). Reduction in size of children's home: Specific guidelines for inauguration [In Japanese]. Memoirs of Hokuriku Gakuin University, Hokuriku Gakuin Junior College(6), $51-63$.

Hayashi, H. (2000). Staff's viewpoints about caring children institutions for children [In Japanese]. Japanese Journal of Social Services, 40(2), 136-151. doi:10.24469/jssw.40.2_136

Hirata, S., \& Negayama, K. (2012). Child protection institutions as allocare systems from the viewpoint of poverty [In Japanese]. Japanese Journal of Developmental Psychology, 23(4), 460-469. doi:10.11201/jjdp.23.460

Iida, M. (2010). Kodomoto tomoni sodachiatte [Growing with children: Three years of smallunit group care; In Japanese]. Kodomoto Fukushi, 3, 7-12.

Ito, K. (2010). Analysis of the quality of life in an institution for maltreated children: Based on interviews with and narratives of children [In Japanese]. Japanese Journal of Social Welfare, 50(4), 82-95. doi:10.24469/jssw.50.4_82

Kato, K. (2003). Issues and prospects of independence facilitation in residential care institutions [In Japanese]. Review of Welfare Society, 3, 78-90.

Kikuchi, Y. (1993). The history and the role of children's homes after World War II: Examining Sukagawa Home [In Japanese]. The Bulletin of Kyoei Gakuen Junior College, 9, 177-188.

Kikuchi, Y., \& Watanabe, T. (1995). Achievements in child care practice in the residential home: from Mrs. Hujino's practice [In Japanese]. The Bulletin of Kyoei Gakuen Junior College, 11, $357-371$.

Kobe, K. (2007). Independence facilitation in residential care institutions: Study of 12 institutions in Gifu [In Japanese]. Journal of Toho Gakuen, 36(1), 35-51.

Ministry of Health, Labour and Welfare. (2019). Shakaiteki yogono suishinni mukete [Toward promotion of out-of-home care].

Mori, S. (Ed.). (2016). 'Shakainiyoru kosodate' jissen Hanbook [Handbook of practicing “child rearing by society"]. Iwasaki Gakujutu Shuppansya. 
International Journal of Child, Youth and Family Studies (2020) 11(4): 80-95

Nagai, R. (2006). The issues concerning the care of abused children in children's homes [In Japanese]. Bulletin of the Japan Lutheran College and Theological Seminary, 39, 89-101.

Nagase, M., \& Karino, H. (2012). Residential care institutions and social exclusion [In Japanese]. Buraku Kaiho, 656, 235-248.

Sato, Y., Sato, S., Yamaguchi, S., \& Furuse, M. (2011). Processes used by staff of Children Protection Institution to Develop Relations with Abused Children [In Japanese]. Journal of Japan Society of Nursing Research, 34(5), 105-114. doi:10.15065/jjsnr.20110729012

Taniguchi, S. (2018). Originality and ingenuity of livelihood support in residential child care [In Japanese]. Bulletin of Aichi Shukutoku University, 8, 63-77.

http://hdl.handle.net/10638/00007949

Yoshimura, Y., \& Yoshimura, M. (2016). Jidoyogoshisetsuno shokibokani tsuite kodomoto shokuinno katarikara kangaeru [Examining the downsizing of children's home by analysing the narratives of the staff and children]. Bulletin of Okazaki Women's Junior College, 49, 8998.

Zhang, Y., Tanaka, E., Anme, T., Mori, S., Bradley, R., \& Lau, J. Y. (2018). Japanese residential care quality and perceived competency in institutionalized adolescents: A preliminary assessment of the dimensionality of care provision. Children and Youth Services Review, 91, 204-212. doi:10.1016/j.childyouth.2018.05.013 